Mitteilungen der Österreichischen Geographischen Gesellschaft, 159. Jg. (Jahresband), Wien 2017, S. 408-412

\title{
Zum Gedenken an eM. O. Univ.-Prof. Dr. Karl Ruppert (1926-2017)
}

\author{
Hugo PENZ, Innsbruck* \\ mit 1 Abb. im Text
}

Mit em. o. Univ.-Prof. Dr. Karl RuPPERT ist am 29. März 2017 ein allseits geschätzter Fachvertreter verstorben, der enge Beziehungen zur österreichischen Geographie gepflegt hat. Er kam am 15. Januar 1926 als Sohn eines Volksschullehrers in Offenbach (Hessen) zur Welt, von dem er das pädagogische Geschick geerbt zu haben scheint. Seine Vorlesungen und Vorträge waren übersichtlich gegliedert und er trug sie in einer klaren, verständlichen Sprache mit fester Stimme vor, wobei der leichte hessische Dialekteinschlag seine Herkunft verriet. Nach einer glücklichen Kindheit erlitt er das Schicksal seiner Generation. Er konnte die Oberrealschule nicht abschließen, sondern musste ein Jahr lang als Luftwaffenhelfer dienen und 1944 zur Wehrmacht einrücken. Am Ende des Krieges geriet er in Italien in amerikanische Gefangenschaft, von der er im Oktober 1945 entlassen wurde. Im Rahmen eines Sonderlehrganges für Heimkehrer legte er im März 1946 die Reifeprüfung ab, sodass er an der Universität Frankfurt am Main die Fächer Mathematik, Physik und Geographie studieren konnte. Dort fand er in Professor Wolfgang HARTKE einen akademischen Lehrer, der ihn

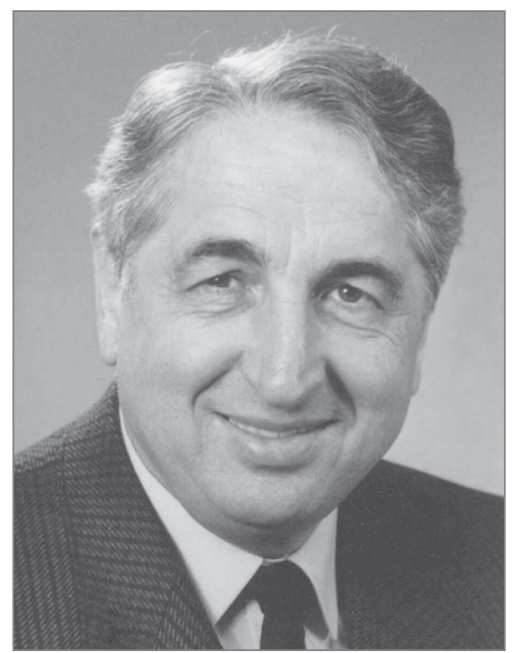

Karl RUPPERT 1926-2017

\footnotetext{
* Tit.a.o. Univ-Prof. Dr. Hugo Penz, Universität Innsbruck, Institut für Geographie, Innrain 52, A-6020 Innsbruck; E-Mail: hugo.penz@uibk.ac.at
} 
anregte und den weiteren Lebenslauf stark beeinflusste. Im Jahre 1950 übertrug ihm das Geographische Institut der Universität Frankfurt a.M. die Stelle einer wissenschaftlichen Hilfskraft, die mit dem Dienst in der Bibliothek verbunden war. 1952 schloss Karl RUPPERT das Studium mit der von Wolfgang Hartke betreuten Dissertation über „Die Leistung des Menschen zur Erhaltung der Kulturböden im südlichen Rheinhessen“ ab.

Im Sommer des Jahres 1952 heiratete er seine aus Groß-Felda (heute Feldatal) am Vogelsberg stammende Frau Irmgard, eine geborene SснміDт (1926-2014), mit der er über 60 Jahre lang eine vorbildliche, durch gemeinsame christliche Werte geprägte Ehe geführt hat. Aus dieser Verbindung ging die Tochter Mechthild, verehelichte Herz hervor, die einen Landwirt aus dem Allgäu heiratete und seither mit ihrer Familie als Ernährungs- und Gesundheitsberaterin in der Gemeinde Wertach/ Allgäu lebt, welche an die österreichische Ortschaft Jungholz angrenzt. Deshalb ließen sich RUPPERT und seine Gattin dort beerdigen. Er und seine Familie fühlten sich in ländlichen Gebirgslandschaften immer wohl. Daher mieteten sie in einem Bauernhof in Lenggries, unmittelbar nördlich der österreichischen Grenze, eine Ferienwohnung, in welcher er über ein halbes Jahrhundert lang häufig die Freizeit verbrachte. Eine stattliche Abordnung bei der Beerdigung zeigte, dass Karl RuPPERT dort sehr gut integriert war.

Als Wolfgang HARTKE 1952 nach München berufen wurde, folgte ihm Karl RUPPERT als wissenschaftlicher Assistent an das Geographische Institut der Technischen Hochschule und wurde im Jahre 1959, nachdem er sich mit einer hervorragenden Arbeit über „Die Bedeutung des Weinbaues und seiner Nachfolgekulturen für die sozialgeographische Differenzierung der Agrarlandschaft in Bayern" habilitiert hatte, zum Privatdozenten ernannt. Nach der Emeritierung von Erich THIEL wurde er 1964 mit der Vertretung des Lehrstuhles für Wirtschaftsgeographie an der Staatswirtschaftlichen Fakultät der Universität München betraut und 1965 an dieser zum Ordinarius ernannt. In der Folgezeit leitete er das Institut bis zu seiner Emeritierung im Jahre 1991 umsichtig und baute es zu einer angesehenen, international bedeutsamen Lehr- und Forschungsstätte aus.

Innerhalb der Fakultät fand Karl RuPPERT rasch Anerkennung. Vor allem Professoren der Betriebswirtschaft, wie der bekannte Wissenschafter Edmund HeInEN (1919-1996), dem die Universität Innsbruck 1974 das Ehrendoktorat verliehen hat, zählten zu seinen Freunden. Obwohl er erst 42 Jahre alt war, wählte ihn das Kollegium in der Zeit der Studentenunruhen für das Studienjahr 1968/1969 zum Dekan der Staatswissenschaftlichen Fakultät. Dabei gelang es ihm durch seine kluge Amtsführung und seine Gesprächsbereitschaft mit allen Gruppen, den Studienbetrieb aufrechtzuerhalten, sodass damals am Wirtschaftsgeographischen Institut alle Lehrveranstaltungen stattfinden konnten. Nach der Bayerischen Hochschulreform wurde die Ludwig-Maximilians-Universität am 1. Oktober 1974 in 21 Fachbereiche gegliedert, die später in Fakultäten umbenannt wurden. Dabei erreichte Karl RUPPERT, dass sein Institut als eigenständige Einheit im Fachbereich Betriebswirtschaft erhalten blieb. Nach der Emeritierung (1991) verfolgte er die weitere Entwicklung mit Interesse, ohne sich in das Institutsgeschehen einzumischen und bedauerte die Auflassung des Diplomstudienganges Wirtschaftsgeographie und die Umgliederung der beiden Professuren vom Department für Betriebswirtschaft in die Fakultät für Geowissenschaften im Jahre 2006.

Während die Lehrveranstaltungen der Wirtschaftsgeographie unter Erich THIEL vorwiegend von Wahlfachstudenten der Staatswirtschaftlichen Fakultät besucht wurden, richtete sich das Angebot unter Karl RUPPERT vermehrt auch an die Studenten der Geographie. Da die meisten der früheren Angestellten bald aus dem Institut ausschieden, war Karl RUPPERT in der glücklichen Lage, junge engagierte Mitarbeiter einstellen zu können. Dabei achtete er neben der fachlichen Qualifikation auch auf die Teamfähigkeit, welche sich bei gemeinschaftlichen Projekten sehr bewährt hat. Nach einem Ruf an die Universität Bonn konnte Karl RUPPERT in den Bleibeverhandlungen 1970/71 die Ausstattung erheblich verbessern. Neben anderen Dienstposten wurde am Institut eine außerordentliche Professur neu geschaffen, die zunächst durch seine Schüler Franz ScHAFFer (1971-1973) und 
Jörg MAIER (1975-1977) und später durch den aus Tübingen berufenen Dozenten Hans-Dieter HAAS (ab 1979) besetzt wurde, welcher ihm im Wintersemester 1992/93 auf dem Lehrstuhl gefolgt ist. Auch die technische Ausstattung konnte laufend verbessert werden. In den Jahren 1978-1981 erhielt das Institut zusätzliche Räume, sodass dieses bei der Emeritierung im Jahre 1991 über eine hervorragende Infrastruktur verfügte.

Karl RUPPERT war immer bestrebt, die Forschungsergebnisse der Öffentlichkeit zugänglich zu machen. Daher begründete er nach der Berufung die Institutsreihe „Münchner Studien zur Sozialund Wirtschaftsgeographie“, in der neben monographischen Beiträgen auch zahlreiche Sammelbände erschienen sind, und im Jahre 1970 ein zweites Publikationsorgan, die „WGI-Berichte zur Regionalforschung“, in welchen verstärkt planungsorientierte Studien veröffentlicht wurden. Sonderhefte innerhalb dieser Reihe waren den Tätigkeitsberichten des Institutes gewidmet.

Der wissenschaftlichen Werdegang Karl RUPPERTs spiegelt die Entwicklung der deutschen Sozialgeographie wider. Bei den Arbeiten regte ihn neben seinem Lehrer Wolfgang HARTKE auch der österreichische Geograph Hans BoBEK stark an. Diese waren seit der gemeinsamen Zeit in Berlin befreundet, in welcher Hans BoвEK als Assistent und Wolfgang HarTKE als Mitarbeiter am Atlas des deutschen Lebensraumes an dem von Norbert KREBS geleiteten Geographischen Institut beschäftigt waren. Während sich andere Humangeographen in den Nachkriegsjahren der Physiognomie und der Genese der Kulturlandschaft widmeten, betrachtete die aufkommende Sozialgeographie diese als „Registrierplatte“ des gesamtgesellschaftlichen Wandels. Dabei erwies sich die Sozialbrache in Süddeutschland, an deren Erforschung Karl RUPPERT maßgeblich beteiligt war, als sehr aussagefähiger Indikator. Auch seine Untersuchungen über den Weinbau in Bayern verfolgten diese Zielsetzung. Ähnliches gilt für seine frühen Studien über die Bedeutung des Fremdenverkehrs. Später entwickelten Karl RUPPERT und sein Schüler Franz SCHAFFER (1968) mit der „,sozialgeographischen Konzeption“ das theoretische Kernstück der „Münchner Schule der Sozialgeographie“, welche die Sozialgeographie nicht als Teilgebiet, sondern als eine für die gesamte Humangeographie relevante Betrachtungsweise ansieht. Das unter der Leitung von Karl RuPPERT als Gemeinschaftspublikation des Institutes erschienene Buch „Sozialgeographie“ (MAIER et al. 1977) fasst diesen Ansatz zusammen und definiert die Sozialgeographie als „Wissenschaft von den räumlichen Organisationsformen und den raumbildenden Prozessen der Daseinsgrundfunktionen menschlicher Gruppen und Gesellschaften“. Dieser Forschungsansatz wurde zwar von manchen theoriegeleiteten Fachkollegen angegriffen, er bildet jedoch nach wie vor eine der wichtigsten Innovationen in der deutschsprachigen Humangeographie, welche vor allem die Schulgeographie und die planungsorientierte Raumforschung nachhaltig beeinflusst hat. An diesem Konzept orientierten sich die meisten Arbeiten des Wirtschaftsgeographischen Institutes der Universität München.

Die ersten Arbeiten von Karl RUPPERT befassten sich mit dem Agrarraum, anschließend widmete er sich als einer der ersten deutschen Geographen intensiv dem Fremdenverkehr, wobei er diesen Terminus später durch den umfassenderen Begriff der Geographie des Freizeitverhaltens ersetzte. Es folgten u.a. Beiträge zu unterschiedlichen Aspekten der Stadtforschung, der Bevölkerungsgeographie und der Regionalentwicklung. Darüber hinaus beschäftigten sich er und seine Schüler auch mit dem Industrialisierungs- und Urbanisierungsprozess sowie mit Fragen des Immobilienmarktes. Bei seinen Untersuchungen war er immer bemüht, eine Verbindung zwischen den theoretischen Erkenntnissen und den Erfordernissen der Praxis herzustellen. Deshalb suchte er frühzeitig Kontakte zur Landesplanung, die dem Institut zahlreiche Forschungsaufträge erteilte. Diese Zusammenarbeit kam den Absolventen zugute, von denen viele in der amtlichen Raumplanung eine Beschäftigung fanden.

Über Jahrzehnte hinweg bekleidete Karl RUPPERT wichtige Funktionen bei bedeutenden wissenschaftlichen Einrichtungen. Er war Ordentliches Mitglied und von 1979 bis 1982 Vizepräsident 
der Akademie für Raumforschung und Landesplanung in Hannover und er engagierte sich in der Landesarbeitsgemeinschaft Bayern dieser Gesellschaft. Daneben wurde er vom Freistaat Bayern in zahlreiche Gremien berufen, und er wirkte dabei u.a. im Landesplanungsbeirat Bayern und in Kommissionen zu Fragen der Verwaltungsgebietsreformen mit. Darüber hinaus wurde er in das Kuratorium für Landschaftspflege und Naturschutz bestellt. Die Österreichische Gesellschaft für Raumforschung und Raumplanung ernannte ihn zum Ehrenmitglied und das Salzburger Institut für Raumforschung zum Korrespondierenden Mitglied. In diesen Funktionen stand er in einem regen Dialog mit den Nachbar- und Planungswissenschaften, und in vielen Gesprächen mit Fachleuten aus der Praxis konnte er wertvolle Erfahrungen sammeln, welche er an seine Studenten und Mitarbeiter weitervermittelte.

Die wissenschaftlichen Forschungen Karl RUPPERTs konzentrierten sich auf drei regionale Schwerpunkte, nämlich Süddeutschland, Südosteuropa und das östliche Mitteleuropa sowie den Alpenraum. Nahezu drei Viertel seiner wissenschaftlichen Veröffentlichungen und die meisten $\mathrm{Zu}$ lassungs-, Diplom- und Doktorarbeiten beschäftigten sich mit dem süddeutschen Raum. Viele Veröffentlichungen entstanden in einer engen Zusammenarbeit mit den Planungsbehörden und bildeten wichtige Bausteine für die Entwicklung der Angewandten Sozialgeographie. Einer breiteren interessierten Öffentlichkeit wurden diese Forschungen durch den in der Reihe der „Wissenschaftliche Länderkunden“ erschienenen Band „Bayern“ zugänglich gemacht (RUPPERT et al. 1987).

Während zahlreiche Geographen seiner Generation mit dem „kommunistischen Osten“ nichts zu tun haben wollten, knüpfte Karl RUPPERT dorthin frühzeitig Kontakte. Seit den 1950er Jahren war er mit dem slowenischen Geographen Vladimir KLEMENČıč eng befreundet, und für viele Kollegen aus dem ehemaligen Jugoslawien und aus dem östlichen Mitteleuropa war er seit den 1960er Jahren ein wichtiger Ansprechpartner. Um den Studenten ein realistisches Bild von den Sozialistischen Staaten zu vermitteln, veranstaltete er dorthin regelmäßig Exkursionen und lud häufig Wissenschafter aus dem Osten an sein Institut ein. Am Ende der 1970er Jahre beteiligte sich Karl RUPPERT am Forschungsprojekt Südosteuropa der Deutschen Forschungsgemeinschaft (DFG), das an der Universität München eingerichtet worden war. Darüber hinaus war er Mitglied und zeitweise Vorsitzender des Wissenschaftlichen Beirates der Münchner Südosteuropa-Gesellschaft. Als Anerkennung für seine herausragenden Leistungen verliehen ihm u.a. die geographischen Gesellschaften von Kroatien, Slowenien und Ungarn die Ehrenmitgliedschaft.

Seit der Übersiedlung nach München (1950) hat sich Karl RUPPERT sehr für den Alpenraum interessiert, wobei zunächst, wie das von der DFG geförderte Symposium in Rottach-Egern im Jahre 1962 zeigt, Fragen der Almwirtschaft im Vordergrund standen. Anschließend regte er zwei Monographien über die Almwirtschaft in den slowenischen und den österreichischen Alpen an. Später befassten sich Karl RUPPERT und seine Schüler verstärkt mit dem Tourismus und der Regionalentwicklung im Alpenraum, auch bevölkerungs-, wirtschafts- und sozialgeographische Themen wurden bearbeitet. Durch seine jahrelangen Beobachtungen bei Fahrten in die Alpen und durch intensive Diskussionen bei Tagungen, die er regelmäßig besuchte, eignete sich Karl RUPPERT neben solchen von Bayern auch hervorragende landeskundliche Kenntnisse in Bezug auf Österreich, Slowenien und Südtirol an. Daher war er mit den vielfältigen Problemen des Alpenraumes sehr gut vertraut und hütete sich vor einseitigen Wertungen. Der österreichische Alpenraum hat mit dem Tod von Karl RUPPERT nicht nur einen hervorragenden Kenner, sondern auch einen Freund verloren.

Deutschland würdigte die vielseitigen Verdienste von Karl RUPPERT durch die Verleihung des Verdienstkreuzes 1. Klasse im Jahre 1997, und die Universität Augsburg ehrte ihn am 27. Juni 2002 mit dem Dr. rer. nat. h. c. für sein Lebenswerk und begründete diese Würdigung mit seiner großen Bedeutung für die Entwicklung der Angewandten Sozialgeographie. Darüber hinaus verlieh ihm die Österreichische Geographische Gesellschaft im Jahre 2006 mit der Franz-von-Hauer-Medaille die höchste Auszeichnung, die sie zu vergeben hat. 


\section{Literaturhinweise}

Maier J., Paesler R., Ruppert K., Schaffer F. (1977), Sozialgeographie (= Das Geographische Seminar). Braunschweig, Westermann.

Ruppert K., GräF P., HeCKL F.X. (1987), Bayern. Eine Landeskunde aus sozialgeographischer Sicht (= Wissenschaftliche Länderkunden, 8/2). Darmstadt, Wissenschaftliche Buchgesellschaft.

Ruppert K., Schaffer F. (1969), Zur Konzeption der Sozialgeographie. In: Geographische Rundschau, 21, S. 205-214.

Schaffer F. (1991), Die Sozialgeographie im Dienste der Öffentlichkeit. Karl Ruppert zum 65. Geburtstag. In: Goppel K., SchafFer F. (Hrsg.), Raumplanung in den 90er Jahren. Festschrift für Karl Ruppert, S. 1-11. Augsburg, Selbstverlag Lehrstuhl für Sozial- und Wirtschaftsgeographie Universität Augsburg. 